\title{
Avaliação da qualidade da colagem de topo da madeira de Cupressus lusitanica Mill. para a produção de painéis colados lateralmente
}

\author{
Quality evaluation of Cupressus Iusitanica Mill. wood \\ end-grain glved joints for edge glued panel production
}

Caio Cesar Faedo de Almeida ${ }^{1}$, Alexsandro Bayestorff da Cunha ${ }^{2}$ e Polliana D'Angelo Rios ${ }^{2}$

\begin{abstract}
Resumo
O objetivo do estudo foi avaliar a qualidade da colagem de dois adesivos em junções de topo na madeira de Cupressus lusitanica visando à produção de painéis de madeira colados lateralmente. A metodologia envolveu o corte de oito árvores de um plantio experimental, as toras obtidas foi realizado o desdobro tangencial para transformação em peças de madeira serrada, que foram secas, classificadas em peças com qualidade superior e resserradas em menores dimensões para a confecção dos corpos de prova. $O$ delineamento experimental relacionou 2 adesivos comumente utilizado na indústria de painéis de madeira, Poliacetato de vinila (PVA) e Emulsão polimérica de isocianato (EPI) em duas gramaturas (180 e 200g/ $\mathrm{m}^{2}$ ) e duas junções (plana e finger-joint), totalizando oito tratamentos. A confecção dos corpos de prova, a execução dos ensaios e a obtenção das variáveis de resistência foram realizadas de acordo com os procedimentos da ASTM 5572 (ASTM, 2005). Os resultados foram submetidos pela análise não paramétrica de Friedman a $95 \%$ de probabilidade. Como resultados, observou-se que: os tratamentos T7 (gramatura $180 \mathrm{~g} / \mathrm{m}^{2}$ ) e T8 (gramatura $200 \mathrm{~g} / \mathrm{m}^{2}$ ), ambos com adesivo EPI e junção de topo tipo finger-joint, apresentaram os melhores valores médios de resistência e porcentagem de falhas na madeira, da mesma forma no atendimento aos parâmetros da ASTM D5572 (ASTM, 2005); o comportamento das junções com adesivo PVA foi comprometida com a aplicação dos pré-tratamentos, especialmente quanto exposto a condições de umidade e temperaturas elevadas, o que prejudicou os resultados.
\end{abstract}

Palavras-chave: Espécie não convencional; Edge Glued Panel; flexão estática; tração paralela.

\begin{abstract}
The aim of the study was to evaluate the bonding quality of two adhesives on top joints of Cupressus lusitanica wood for edge glued panel production. The methodology involved cutting eight trees of an experimental planting; the obtained logs were submitted to tangential sawing for processing in lumber, dried, classified in pieces with superior quality and re-sawing to smaller pieces to specimen production. The experimental design used 2 adhesives, Polyacetate vinyl (PVA) and Emulsion of polymeric isocyanate (EPI), two grammages (180 and $200 \mathrm{~g} / \mathrm{m}^{2}$ ) and two joints (flat, finger-joint), totaling eight treatments. The preparation of the specimens, testing procedure and the resistance variables were carried out according to the procedures of ASTM 5572 (2005). The results were analyzed by non-parametric analysis of Friedman at $95 \%$ probability. As a result, we observed that: treatments T7 (grammage $180 \mathrm{~g} / \mathrm{m}^{2}$ ) and T8 (grammage $200 \mathrm{~g} / \mathrm{m}^{2}$ ), both with adhesive EPI and the top junction type finger-joint, showed better mean values of resistance and percentage of failures in the wood. While obeying the parameters of ASTM D5572 (2005); the behavior of joints with PVA adhesive was influenced by pre-treatments, especially when exposed to humid conditions and high temperature, which affected the results.
\end{abstract}

Keywords: Non-conventional species; Edge glued panel; static bending; parallel tension.

\section{INTRODUÇÃO}

Nos últimos anos o setor de base florestal apresentou um aumento na demanda por madeira, causado principalmente por estímulos governamentais ao setor de móveis, que beneficiou as indústrias de painéis de madeira, uma vez que a maior parcela da produção da indústria moveleira brasileira é baseada em painéis de madeira reconstituída ou processada mecanicamente (GALINARI et al., 2012).

${ }^{1}$ Mestre em Engenharia Florestal. UDESC - Universidade do Estado de Santa Catarina. Av. Luiz de Camões, 2090 -88520000 - Lages, SC, Brasil. E-mail: caio-almeida@florestal.eng.br

${ }^{2}$ Professor(a) Titular(a) do Departamento de Engenheria Florestal. Universidade do Estado de Santa Catarina. Av. Luiz de Camões, 2090 -88520-000 - Lages, SC, Brasil. E-mail: alexsandro.cunha@udesc.br; polliana.rios@udesc.br

Sci. For., Piracicaba, v. 45, n. 113, p. 9-19, mar. 2017 DOI: dx.doi.org/10.18671/scifor.v45n113.01 
A indústria de painéis de madeira utiliza principalmente matéria-prima de baixa massa específica, dado que espécies com esta característica apresentam melhor qualidade de colagem pela melhor acomodação entre lâminas e partículas da madeira, quando submetido à prensagem (IWAKIRI et al. 2012a). Desta forma, as espécies do gênero Pinus destacam-se na indústria de painéis, tanto para uso estrutural ou não estrutural.

Para atender esta demanda de matéria-prima cada vez mais crescente, torna-se necessário não somente aumentar as áreas de plantio com espécies atualmente utilizadas, mas também buscar opções de outras espécies de rápido crescimento que possam contribuir de forma quantitativa e qualitativa para suprir as necessidades das indústrias, bem como, contribuir para o desenvolvimento e geração de novos produtos (TRIANOSKI et al., 2014).

Neste sentido, estão sendo realizadas pesquisas com diversas espécies (IWAKIRI et al., 2012b) de coníferas, como a Cryptomeria japonica e o Cupressus lusitanica (PEREIRA; HIGA, 2003; PINTO et al., 2011; CARNEIRO et al., 2009; TRIANOSKI et al., 2013). Para as condições da região do planalto catarinense o C. lusitanica mostra-se adequado para o cultivo, contudo, a espécie ainda é utilizada apenas como cerca-viva e quebra-vento (OKINO et al., 2008; SHIMIZU et al., 1995, SHIMIZU et al., 2006).

O C. lusitanica é uma gimnosperma pertencente à família Cupressaceae, originária das regiões montanhosas do México, Guatemala, El Salvador e Honduras (CHAVES; FONSECA, 1991), apresentando incremento de até $36 \mathrm{~m}^{3} /$ ha.ano (SHIMIZU et al., 1995). A madeira da espécie demonstra ser adequada para múltiplos usos como madeira sólida, painéis de madeira e celulose e papel (PEREIRA; HIGA, 2003; OKINO et al., 2010; XAVIER et al., 2012).

A madeira de C. lusitanica foi avaliada quanto às propriedades físicas, químicas, anatômicas (PEREIRA; HIGA, 2003), durabilidade natural (OKINO et al., 2008) e ao rendimento em madeira serrada (ROQUE et al., 2010). Entretanto para a espécie, são escassas informações referentes ao comportamento quando utilizada para produtos colados de madeira sólida.

Dentre estes, os produtos de maior valor agregado (PMVA) podem apresentar informações relevantes quanto à interação madeira-adesivo, pois destacam-se por utilizar peças de madeira sólida coladas em junções de topo e laterais, em especial os painéis colados lateralmente (Edge Glued Panel - EGP) (MAYER, 2007; PEREIRA; HIGA, 2003).

O objetivo do presente estudo foi avaliar a qualidade da colagem de dois adesivos (Poliacetato de vinila - PVA e Emulsão polimérica de isocianato - EPI) em junções de topo na madeira de Cupressus lusitanica visando a produção de painéis de madeira colados lateralmente (Edge Glued Panel - EGP).

\section{MATERIAL E MÉTODOS}

\section{Matéria-prima}

A madeira de C. lusitanica utilizada no estudo foi proveniente de um plantio experimental com 19 anos e área de 72,21 ha, pertencente à Empresa Florestal Gateados Ltda de Campo Belo do Sul SC. Foram utilizadas oito árvores com altura total média de 19,7 m, altura comercial média de 15,2 $\mathrm{m}$, diâmetro à altura do peito (DAP) variando entre 20 e $37 \mathrm{~cm}$ e massa específica básica de 0,396 $\mathrm{g} / \mathrm{cm}^{3}$. De cada árvore, foram retiradas seis toras com comprimento de 1,90 m para a produção dos corpos de prova e outros estudos do laboratório. As toras foram desdobradas tangencialmente na Empresa Boa Esperança Indústria, Comércio e Exportação de Madeiras Ltda., localizada no município de Capão Alto - SC. O desdobro proporcionou a obtenção de 700 peças de madeira serrada com dimensões de 1,90 m de comprimento, $140 \mathrm{~mm}$ de largura e $15 \mathrm{~mm}$ de espessura. As peças foram secas ao ar livre até a umidade de equilíbrio da região $( \pm 15 \%)$ e classificadas visualmente de acordo com a presença de defeitos naturais e de processamento, de modo que as peças com qualidade superior (Clear Blocks) foram utilizadas para a confecção dos painéis colados.

\section{Delineamento experimental}

O delineamento experimental foi desenvolvido no esquema fatorial $2 \times 2 \times 2$, relacionando 2 adesivos (poliacetato de vinila - PVA e emulsão polimérica de isocianato - EPI), duas gramaturas $\left(180\right.$ e $\left.200 \mathrm{~g} / \mathrm{m}^{2}\right)$ e duas junções (plana e finger-joint), como apresentado na Tabela 1. 
Tabela 1. Delineamento experimental para a avaliação da qualidade da colagem.

Table 1. Experimental design for the evaluation of gluing quality.

\begin{tabular}{lccc}
\hline Tratamento & Adesivo & Gramatura $\left(\mathbf{g} / \mathbf{m}^{\mathbf{2}}\right)$ & Junção de topo \\
T1 & PVA & 180 & Plana \\
T2 & PVA & 200 & Plana \\
T3 & PVA & 180 & Finger-joint \\
T4 & PVA & 200 & Finger-joint \\
T5 & EPI & 180 & Plana \\
T6 & EPI & 200 & Plana \\
T7 & EPI & 180 & Finger-joint \\
T8 & EPI & 200 & Finger-joint \\
\hline
\end{tabular}

A confecção dos corpos de prova e a execução dos ensaios foram realizadas de acordo com os procedimentos da ASTM 5572 (ASTM, 2005). A avaliação da qualidade das juntas coladas nos oito tratamentos foi realizada por meio da determinação da resistência máxima a flexão estática e a tração paralela.

\section{Corpos de prova}

As peças de madeira serrada da espécie utilizadas no experimento enquadram-se na classe de resistência C25 para coníferas, segundo a norma NBR 7190 (ABNT, 1997), determinação esta realizada em trabalhos anteriores à produção de painéis colados lateralmente.

Onde estas peças foram classificadas para a confecção dos corpos de prova, sendo destopadas em peças livres de defeitos (Clear Blocks) em dimensões padronizadas pela norma de referência para cada ensaio.

A confecção dos corpos de prova para o ensaio de flexão estática envolveu a utilização de 500 peças destopadas em $150 \mathrm{~mm}$ de comprimento, enquanto que para o ensaio de tração, o destopo originou 500 peças com $110 \mathrm{~mm}$ de comprimento. Salienta-se que em ambos os casos, não houve o corte longitudinal das peças (refilo), ou seja, as peças continuavam com $140 \mathrm{~mm}$ de largura.

Para a avaliação das juntas planas e finger-joint pelos ensaios de flexão estática e tração, houve a necessidade do fresamento das peças para a obtenção da segunda junção mencionada. $O$ fresamento foi realizado na unidade de beneficiamento da Empresa MADEPAR Indústria e Comércio de Madeiras Ltda., Lages - SC, sendo que a face fresada apresentava 5 dentes com 7,15 mm de comprimento, 2,40 $\mathrm{mm}$ de largura na base e 1,60 $\mathrm{mm}$ de largura na ponta.

Após a obtenção das peças com topos planos e fresados, as peças foram armazenadas por aproximadamente 60 dias em câmara com temperatura e umidade controladas, para uniformização do teor de umidade em $12 \%$.

Após este período, foi realizada a aplicação das resinas nas duas gramaturas destacadas no delineamento. Na sequência, as duas peças de cada corpo de prova foram unidas manualmente e encaminhadas para a prensa hidráulica, com carga de $3.000 \mathrm{kgf}$ aplicados sobre $112,50 \mathrm{~cm}^{2}$, o que corresponde à pressão de 2,61 MPa por 180 minutos para a resina PVA e 30 minutos para a EPI, como recomendado pelo fabricante. Após a prensagem, as peças foram mantidas em ambiente climatizado por aproximadamente 30 dias para garantir a cura completa dos adesivos e a estabilização do teor de umidade das peças. Após o período de climatização, as peças foram refiladas em serra fita simples para as dimensões requeridas pelos ensaios de flexão estática e tração, sendo posteriormente armazenadas até o momento da aplicação dos pré-tratamentos e dos ensaios mecânicos.

De acordo com a norma, os corpos de prova para flexão estática deveriam apresentar dimensões de 12,8 X 19,2 X 307,2 mm em espessura, largura e comprimento, respectivamente, com vão de 24 vezes a altura do corpo de prova. No entanto, em função das peças de madeira serrada de $C$. lusitanica apresentarem $15 \mathrm{~mm}$ de espessura e fresagem na horizontal, foi necessário a adaptação das dimensões para 10 X 15 X 240 mm. Entretanto, a norma ASTM 5572 (ASTM, 2005) descreve a necessidade de acréscimo de 50,8 $\mathrm{mm}$ ao comprimento total para garantir a estabilidade do corpo de prova apoiado sobre os dois pontos de reação inferiores, como demonstra a Figura 1. 


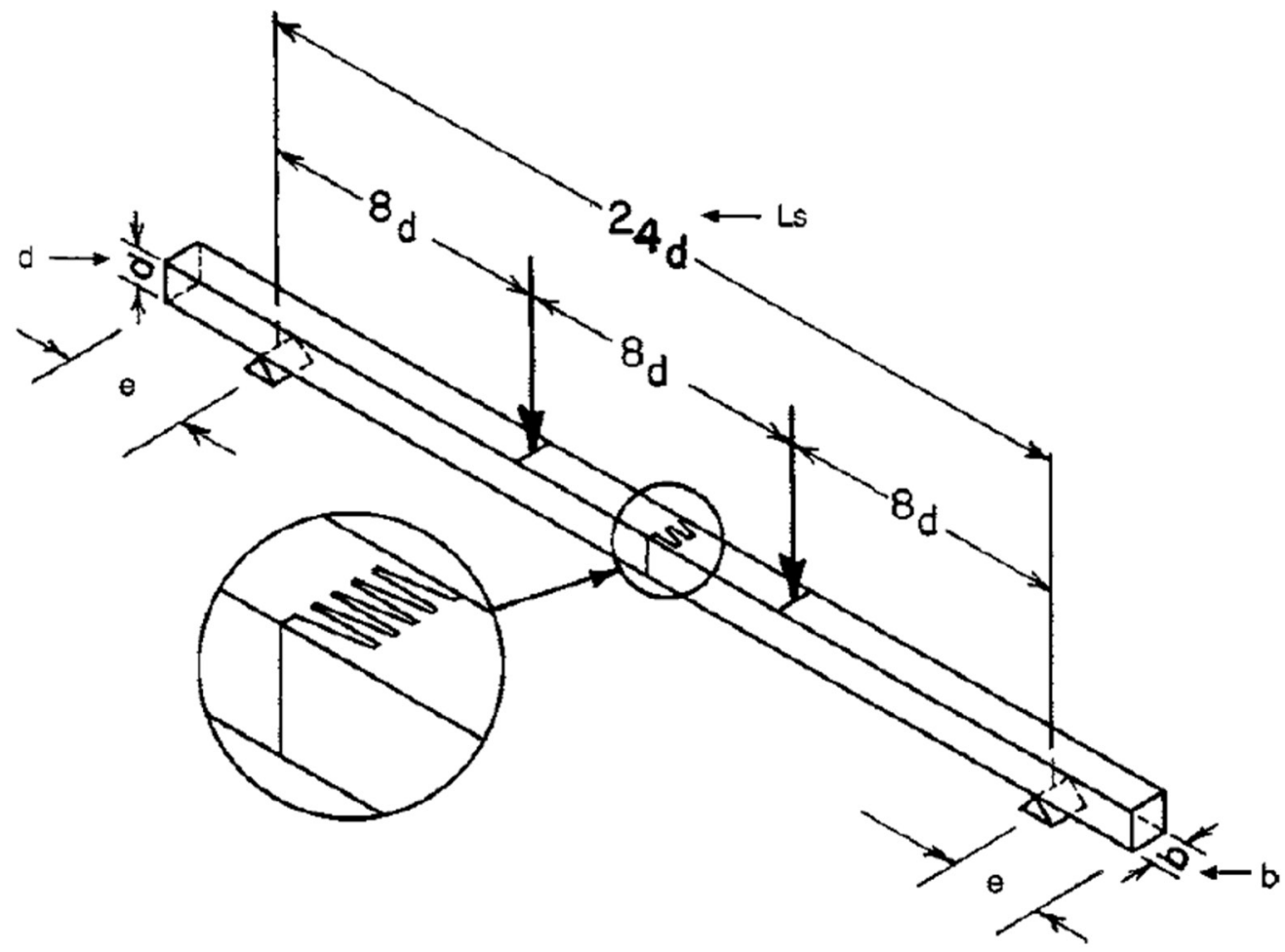

Fonte: ASTM 5572 (ASTM, 2005)

Ls: Comprimento total entre apoios; b: largura; d: espessura; e: comprimento extra.

Figura 1. Geometria de corpo de prova e modo de realização de ensaio de flexão estática. Figure 1. Specimen geometry and static bending test form execution.

Assim como para os corpos de prova de flexão estática, a norma ASTM 5572 (ASTM, 2005) determina as dimensões preferenciais para os corpos de prova do ensaio de tração paralela de 6,35 x 19,05 x $254 \mathrm{~mm}$ em espessura, largura e comprimento. Contudo, da mesma forma que para a o ensaio de flexão estática, foi necessário a alteração da geometria dos corpos de prova para 5,00 x 15,0 x 195,5 mm em largura, espessura e comprimento. Entretanto, cabe salientar que foram mantidas as relações dimensionais especificadas em norma como demonstra a Figura 2.

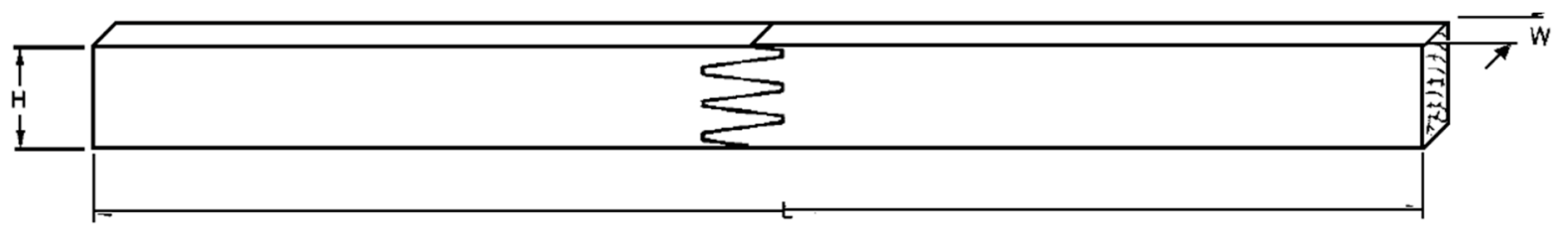

ASTM 5572 (ASTM, 2005).

L: Comprimento total entre apoios; w: largura; h: espessura.

Figura 2. Geometria dos corpos de prova do ensaio de tração.

Figure 2. Specimen geometry fort traction test.

\section{Pré-tratamentos}

O objetivo das juntas coladas com PVA e EPI era a formação de painéis EGP para a indústria moveleira, ou seja, ambiente interno em condição seca. Desta forma, foi necessário a aplicação de 4 pré-tratamentos, totalizando 80 corpos de prova por tratamento para o ensaio de flexão estática e 80 corpos de prova por tratamento para o ensaio de tração paralela. Os pré-tratamentos aplicados foram:

a) Teste seco (P1): condição ideal de uso do painel EGP, visando à obtenção da máxima resistência das junções coladas após a cura total do adesivo.

b) Teste Úmido (P2): pior condição de uso do painel. O teste consistiu na aplicação de três ciclos de umedecimento e secagem, no qual, os corpos-de-prova foram imersos em água à temperatura ambiente durante 4 horas. Em seguida os corpos de prova foram retirados da água e acondiciona- 
dos em estufa com circulação forçada de ar à uma temperatura de $41 \pm 3^{\circ} \mathrm{C}$ durante um período de 19 horas. Após os ciclos foram realizados os ensaios.

c) Teste de elevada temperatura (P3): degradação térmica dos adesivos por elevada temperatura, onde, o pré-tratamento consistiu em acondicionar os corpos de prova em estufa com circulação forçada de ar à uma temperatura de $104 \pm 3^{\circ} \mathrm{C}$ durante um período de 6 horas e imediatamente em seguida realizar os ensaios.

d) Teste de elevada temperatura e umidade (P4): degradação dos adesivos durante o período de transporte. Através da aplicação de duas camadas de filme plástico para evitar que a umidade dos corpos de prova seja perdida quando aquecidos à temperatura de $65 \pm 1^{\circ} \mathrm{C}$ em estufa por 20 minutos, seguido de ensaio realizado em ambiente climatizado à temperatura de $24 \pm 1^{\circ} \mathrm{C}$.

\section{Ensaios de flexão estática e tração}

Os ensaios mecânicos foram realizados em máquina universal de ensaios, EMIC modelo DL$-300 \mathrm{kN}$, com velocidade $12,7 \mathrm{~mm}$ por minuto. Os resultados obtidos foram o módulo de ruptura (MOR) no ensaio de flexão estática e a tensão máxima no ensaio de tração paralela. Ainda foi determinado o percentual de falha na madeira (FM\%) pela análise visual conforme descrito em norma.

O percentual de falha na madeira das junções foi determinado visualmente seguindo a metodologia descrita para junções finger-joint na norma ASTM D5572 (ASTM, 2005) tanto para tração quanto para flexão (sem requisito da norma), onde existem seis níveis de classificação, como apresenta a Figura 3., sendo:

Nível 1: correspondente ao rompimento principalmente no adesivo e com menos de $70 \%$ de falha na madeira, os seguintes níveis 2 a 6 genericamente à valores acima de $70 \%$, contudo, diferenciando na forma como ocorre o rompimento do corpo de prova, o qual determina o nível atingido e indica melhor eficiência de colagem, sendo:

Nível 2: falha ao longo da linha de cola, mas com falha na madeira superior à 70\%;

Nivel 3: falha ao longo do perfil dos dentes, com remoção de alguns dentes ou partes destes;

Nível 4: falha na base dos dentes com remoção parcial ou total destes;

Nível 5: falha iniciada nos dentes e propagada ao longo do corpo de prova;

Nível 6: falha totalmente no corpo de prova, não influenciada pela junção.
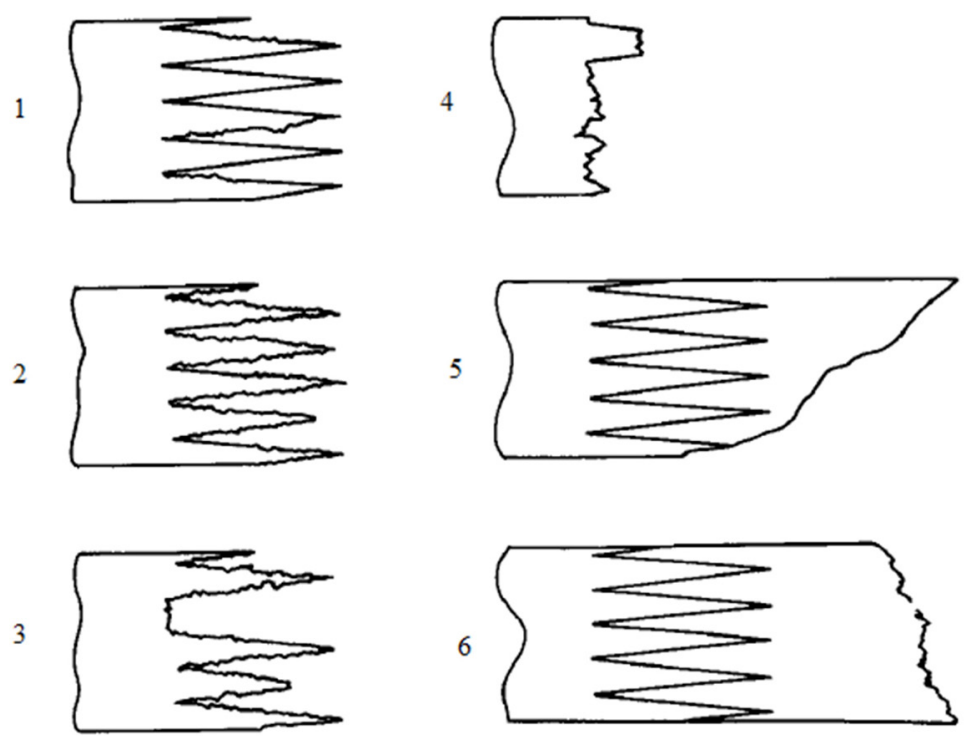

ASTM 5572 (ASTM, 2005).

I: Nível I de falha na madeira; 2: Nível 2 de falha na madeira; 3: Nível 3 de falha na madeira; 4: Nível 4 de falha na madeira; 5: Nível 5 de falha da madeira; 6: Nível 6 de falha na madeira.

Figura 3. Índices de classificação visual de falha na madeira de acordo com a norma ASTM 5572 (ASTM, 2005). Figure 3. Wood failure visual classification indexes according to ASTM 5572 (2005).

\section{Análise dos dados}

Os resultados encontrados foram submetidos preliminarmente ao teste de Kolmogorov-Smirnov para a verificação da normalidade dos dados e ao teste de Levene para a homogeneidade das variân- 
cias. Como os pressupostos não foram atingidos, foi realizada a análise não paramétrica pelo teste de Friedman a 95\% de probabilidade, tendo em vista que o objetivo era a determinação do melhor tratamento, independentemente do tipo de adesivo, quantidade de adesivo aplicado (gramatura) e tipo de junta de topo a ser colada.

\section{RESULTADOS E DISCUSSÃO}

\section{Avaliação da junção de topo pelo ensaio de flexão estática}

A Tabela 2 apresenta os valores médios obtidos para MOR após a aplicação dos pré-tratamentos determinados na norma de referência. Para a condição seca (P1), o tratamento que apresentou maior valor médio de foi o T7 (EPI, 180, finger) com 22,11 MPa de resistência, o qual foi equivalente estatisticamente ao T8 (EPI, 200, finger) e ao T4 (PVA, 200, finger), enquanto todos os tratamentos com junção plana apresentaram resultados com os menores valores médios.

Tabela 2. Valores médios para módulo de ruptura (MOR) à flexão estática (MPa).

Table 2. Mean values for rupture modulus of static bending.

\begin{tabular}{|c|c|c|c|c|c|c|c|c|}
\hline Trat. & P1 (MPa) & FM & P2 (MPa) & FM & P3 (MPa) & FM & P4 (MPa) & FM \\
\hline 1 & $\begin{array}{l}06,00 d \\
(32,92)\end{array}$ & $\begin{array}{c}1 \\
(1-5)\end{array}$ & $\begin{array}{l}00,00 \mathrm{c} \\
(00,00)\end{array}$ & $\begin{array}{c}1 \\
(1-1)\end{array}$ & $\begin{array}{l}05,03 c \\
(38,75)\end{array}$ & $\begin{array}{c}1 \\
(1-1)\end{array}$ & $\begin{array}{c}05,34 \mathrm{~cd} \\
(46,15)\end{array}$ & $\begin{array}{c}1 \\
(1-1)\end{array}$ \\
\hline 2 & $\begin{array}{l}03,92 d \\
(33,00)\end{array}$ & $\begin{array}{c}1 \\
(1-1) \\
\end{array}$ & $\begin{array}{c}00,00 c \\
(00,00)\end{array}$ & $\begin{array}{c}1 \\
(1-1) \\
\end{array}$ & $\begin{array}{r}08,84 c \\
(32,80)\end{array}$ & $\begin{array}{c}1 \\
(1-1) \\
\end{array}$ & $\begin{array}{l}04,08 d \\
(42,97)\end{array}$ & $\begin{array}{c}1 \\
(1-1) \\
\end{array}$ \\
\hline 3 & $\begin{array}{c}14,50 \mathrm{bc} \\
(21,37)\end{array}$ & $\begin{array}{c}1 \\
(1-2) \\
\end{array}$ & $\begin{array}{l}09,17 b \\
(24,19)\end{array}$ & $\begin{array}{c}1 \\
(1-3) \\
\end{array}$ & $\begin{array}{c}16,98 \mathrm{ab} \\
(23,39)\end{array}$ & $\begin{array}{c}1 \\
(1-5)\end{array}$ & $\begin{array}{c}09,16 c \\
(36,58)\end{array}$ & $\begin{array}{c}1 \\
(1-3) \\
\end{array}$ \\
\hline 4 & $\begin{array}{c}15,43 a b \\
(15,89)\end{array}$ & $\begin{array}{c}1 \\
(1-2)\end{array}$ & $\begin{array}{l}11,41 \mathrm{ab} \\
(24,51)\end{array}$ & $\begin{array}{c}2 \\
(1-2)\end{array}$ & $\begin{array}{c}19,30 a b \\
(21,85)\end{array}$ & $\begin{array}{c}1 \\
(1-1)\end{array}$ & $\begin{array}{c}08,11 \mathrm{~cd} \\
(33,54)\end{array}$ & $\begin{array}{c}1 \\
(1-5)\end{array}$ \\
\hline 5 & $\begin{array}{c}08,37 \mathrm{~cd} \\
(27,50)\end{array}$ & $\begin{array}{c}1 \\
(1-1)\end{array}$ & $\begin{array}{c}10,63 a b \\
(32,88)\end{array}$ & $\begin{array}{c}1 \\
(1-1)\end{array}$ & $\begin{array}{l}12,78 \mathrm{bc} \\
(30,61)\end{array}$ & $\begin{array}{c}1 \\
(1-1)\end{array}$ & $\begin{array}{l}11,03 \mathrm{bc} \\
(25,94)\end{array}$ & $\begin{array}{c}1 \\
(1-1)\end{array}$ \\
\hline 6 & $\begin{array}{l}07,08 \mathrm{~cd} \\
(22,98)\end{array}$ & $\begin{array}{c}1 \\
(1-1)\end{array}$ & $\begin{array}{l}07,74 b \\
(29,18)\end{array}$ & $\begin{array}{c}1 \\
(1-2)\end{array}$ & $\begin{array}{l}10,99 \mathrm{bc} \\
(29,50)\end{array}$ & $\begin{array}{c}1 \\
(1-1)\end{array}$ & $\begin{array}{c}08,53 \mathrm{~cd} \\
(28,48)\end{array}$ & $\begin{array}{c}1 \\
(1-1)\end{array}$ \\
\hline 7 & $\begin{array}{l}22,11 a \\
(11,15)\end{array}$ & $\begin{array}{c}3 \\
(1-5)\end{array}$ & $\begin{array}{l}20,43 a \\
(24,01)\end{array}$ & $\begin{array}{c}3 \\
(1-5)\end{array}$ & $\begin{array}{l}22,92 a \\
(24,36)\end{array}$ & $\begin{array}{c}3 \\
(1-5)\end{array}$ & $\begin{array}{r}20,57 \mathrm{ab} \\
(29,57)\end{array}$ & $\begin{array}{c}2 \\
(1-5)\end{array}$ \\
\hline 8 & $\begin{array}{l}17,45 a b \\
(17,55)\end{array}$ & $\begin{array}{c}2 \\
(1-5)\end{array}$ & $\begin{array}{l}21,14 a \\
(27,45)\end{array}$ & $\begin{array}{c}3 \\
(1-5)\end{array}$ & $\begin{array}{l}23,50 a \\
(10,92)\end{array}$ & $\begin{array}{c}2 \\
(1-5)\end{array}$ & $\begin{array}{l}20,85 a \\
(18,99)\end{array}$ & $\begin{array}{c}2 \\
(1-4)\end{array}$ \\
\hline
\end{tabular}

Trat.: tratamento, PT: pré-tratamento seco, P2: pré-tratamento úmido, P3: pré-tratamento alta temperatura, P4: pré-tratamento a elevada temperatura e umidade. Médias de pré-tratamentos representam módulo de ruptura (MOR) expresso em MPa; Valores entre parênteses nos pré-tratamentos representam o coeficiente de variação em porcentagem; FM: nível de classificação de porcentagem de falha na madeira. Valores entre parênteses no FM representam os níveis mínimos e máximos. Médias seguidas pela mesma letra na coluna não diferem estatisticamente entre si pelo teste de Friedman ao nível de $95 \%$ de probabilidade.

Em relação à norma de referência, ASTM D5572 (ASTM, 2005), que define como resistência mínima 13,8 MPa, verifica-se que somente os tratamentos com junção finger-joint (T3, T4, T7 e T8), independente do adesivo e da gramatura utilizados, foram os únicos a atingir o parâmetro mencionado. Contudo, apenas os tratamentos T7 e T8 (ambos com adesivo EPI e junção finger-joint) atenderam ao requisito da norma quanto ao percentual de falha na madeira (60\%), apresentando valores superiores com médias 3 e 2, respectivamente. Assim, os resultados obtidos corroboram com Follrich et al. (2010), que descrevem que o aumento na área superficial (plana para finger) na interação entre adesivo e madeira proporciona uma maior resistência da junção, principalmente quando combinado com uma madeira e um adesivo que apresentem boas propriedades de adesão.

Em relação a outros estudos disponíveis, tem-se Prata (2010) que estudou cinco espécies de pinus tropicais com adesivo PVA, encontrando valores médios de 41,16 MPa para Pinus tecunumanii, 33,19 MPa para P. taeda, 40,03 MPa para P. oocarpa, 38,58 MPa para P. maximinoi, 35,38 para P. caribaea var. hondurensis e 35,34 MPa para P. chiapensis, os quais foram muito superiores ao do presente estudo. Os valores médios do presente estudo também foram inferiores ao reportado por Vrazel e Sellers (2004), que observaram para MOR no ensaio de flexão estática 74,09 MPa para madeira de Pinus spp. e 68,42 MPa para madeira de Pseudotsuga menziesii.

Acredita-se que a superioridade dos valores encontrados pelos autores supracitados se deve a maior geometria, comprimento dos dentes das junções finger-joint e a maior massa específica das madeiras em relação à do C. lusitanica $\left(0,396 \mathrm{~g} / \mathrm{cm}^{3}\right)$, tendo em vista que Vick (1999) cita que com 
o aumento da densidade da madeira, ocorre um aumento da resistência à ruptura da madeira, ou seja, maior qualidade da ligação. O autor cita ainda que o incremento de resistência é obtido até $0,75 \mathrm{~g} / \mathrm{cm}^{3}$, sendo que após este valor, a resistência tende a decrescer em função da dificuldade de penetração do adesivo.

Soma-se a superioridade dos valores médios do segundo trabalho (VRAZEL; SELLERS, 2004), a qualidade do adesivo poliuretano em relação ao PVA e ao EPI, o que torna a junção mais rígida e resistente.

Na avaliação dos resultados após a aplicação do pré-tratamento úmido (P2), fica evidenciado a redução dos valores de MOR para os tratamentos compostos com adesivo PVA em relação à condição seca. Comportamento semelhante também foi observado por Clau et al. (2011), com destaque para os tratamentos constituídos por junções planas.

Sobre a superioridade dos tratamentos, verifica-se que os melhores foram o T8 (EPI, 200, finger) com 20,43 MPa de resistência, T7 (EPI, 180, finger) com 20,43 MPa, e o T4 (PVA, 200, finger) com $11,41 \mathrm{MPa}$, tendo em vista que foram equivalentes estatisticamente $\mathrm{e}$ foram os únicos a atingirem o requisito mínimo de falha na madeira. Já os piores tratamentos foram o T1 (PVA, 180, plana) e o T2 (PVA, 200, plana) que não chegaram a ser testados em função de descolarem durante a aplicação do pré-tratamento. Este comportamento dos tratamentos T1 e T2 é devido à solubilização do adesivo PVA em ambiente aquoso.

Os valores médios encontrados após a aplicação do pré-tratamento 3 (P3) demonstram mais uma vez a superioridade e equivalência estatística dos tratamentos T8 (EPI, 200, finger), T7 (EPI, 180, finger), T4 (PVA, 200, finger) e também do T3 (PVA, 180, finger), de modo que somente estes quatro tratamentos atenderam ao especificado pela norma (13,8 MPa), não havendo requisito mínimo de falha na madeira para esta condição.

Todos os tratamentos compostos pelas junções planas, independente do adesivo e da gramatura foram equivalentes estatisticamente e apresentaram resultados pouco expressivos para o ensaio realizado.

O comportamento dos tratamentos T1 (PVA, 180, plana) e T2 (PVA, 200, plana), corrobora com a afirmação de Clau et al. (2011), que diz que o adesivo PVA apresenta proeminente degradação térmica a partir de $50^{\circ} \mathrm{C}$ e tem suas propriedades adesivas seriamente comprometidas, inviabilizando a utilização como elemento ligante entre peças de madeira colada. Os autores destacam também, que a partir da referida temperatura, o percentual de falha na madeira reduz para aproximadamente $0 \%$, devido ao comportamento termoplástico do adesivo.

Entretanto para os tratamentos compostos por adesivo PVA e junções finger-joint (T3 e T4) não foi observado tal comportamento, onde, o pré-tratamento P3 apresentou médias superiores às observadas para P1, que é considerada a condição de uso ideal. Este resultado pode ser devido ao curto período de exposição à elevada temperatura, a qual pode promover a movimentação do adesivo $e$ consequente melhor penetração na estrutura da madeira e resistência da junta colada.

Para o adesivo EPI, o presente estudo apresentou valores superiores ao observado por Clau et al. (2011) para Fagus sylvatica com diferentes adesivos, tendo em vista que o autor encontrou 10,82 MPa para EPI, 10,86 MPa para poliuretano, 10,53 MPa para melamina formaldeído, 11,48 MPa para uréia formaldeído e 8,75 MPa para melamina uréia formaldeído em temperatura de $100^{\circ} \mathrm{C}$. Já em relação ao estudo de Prata (2010), os valores médios foram inferiores, tendo em vista que o autor encontrou intervalo de resistência para as cinco espécies de pinus tropicais valores entre $49 \mathrm{MPa} e$ 33,76 MPa; a razão para a diferença já foi mencionada anteriormente no ensaio na condição seca.

A análise dos resultados com aplicação do pré-tratamento P4 corrobora com a afirmação de Follrich et al. (2011) que citam que para adesivo PVA ocorre uma degradação química na linha de cola, em função da hidrólise à elevadas temperaturas em combinação com a umidade do ambiente. Assim, denota-se que as características químicas do adesivo tem papel fundamental para a resistência a hidrólise, especialmente a elevadas temperaturas. Porém, para os corpos de prova colados com adesivo EPI, observou-se um relativo aumento de resistência quando submetidos a temperatura e umidade moderadas, com exceção do tratamento T7 (EPI, 180, finger).

Assim, após a aplicação do pré-tratamento P4, observa-se que os tratamentos compostos por EPI foram superiores ao requisito de 6,9 $\mathrm{MPa}$, descrito para a pior condição de teste. Entre os tratamentos de PVA apenas se adequaram ao requisito os tratamentos T3 (PVA, 180, finger) e T4 (PVA, 200, finger).

Sci. For., Piracicaba, v. 45, n. 113, p. 9-19, mar. 2017 DOI: dx.doi.org/10.18671/scifor.v45n113.01 


\section{Avaliação da junção de topo pelo ensaio de tração paralela}

Os valores médios de tensão máxima no ensaio de tração paralela, que estão apresentados na Tabela 3, juntamente com os coeficientes de variação e a porcentagem de falhas na madeira, demonstraram que independente do pré-tratamento aplicado, os tratamentos T7 (EPI, 180, finger) e T8 (EPI, 200, finger) apresentaram os melhores resultados e foram estatisticamente iguais, enquanto que as uniões coladas com junção plana foram estatisticamente inferiores aos tratamentos análogos constituídos por junções do tipo finger-joint.

Cabe salientar que as dimensões dos corpos de prova utilizados para o ensaio de tração paralela foram menores quando comparado aos do ensaio de flexão estática, o que reflete em uma menor área de contato entre madeira e adesivo e consequentemente maior exposição e degradação das linhas de cola à condição ambiental simulada pelos pré-tratamentos.

Tabela 3. Valores médios de tensão máxima à tração paralela (MPa).

Table 3. Mean values for maximum tension to parallel tension.

\begin{tabular}{|c|c|c|c|c|c|c|c|c|}
\hline Trat. & P1 & FM & P2 & $F M$ & P3 & FM & P4 & FM \\
\hline 1 & $\begin{array}{l}03,03 d \\
(29,29)\end{array}$ & $\begin{array}{c}1 \\
(1-1)\end{array}$ & $\begin{array}{l}00,00 c \\
(00,00)\end{array}$ & $\begin{array}{c}1 \\
(1-1)\end{array}$ & $\begin{array}{l}02,67 e \\
(34,35)\end{array}$ & $\begin{array}{c}1 \\
(1-1)\end{array}$ & $\begin{array}{l}02,73 e \\
(36,89)\end{array}$ & $\begin{array}{c}1 \\
(1-1)\end{array}$ \\
\hline 2 & $\begin{array}{l}04,70 d \\
(26,42)\end{array}$ & $\begin{array}{c}1 \\
(1-1)\end{array}$ & $\begin{array}{l}00,00 \mathrm{c} \\
(00,00)\end{array}$ & $\begin{array}{c}1 \\
(1-1)\end{array}$ & $\begin{array}{c}04,50 \mathrm{de} \\
(32,13)\end{array}$ & $\begin{array}{c}1 \\
(1-1)\end{array}$ & $\begin{array}{c}03,42 \text { de } \\
(29,46)\end{array}$ & $\begin{array}{c}1 \\
(1-1)\end{array}$ \\
\hline 3 & $\begin{array}{c}09,04 \mathrm{bc} \\
(10,25)\end{array}$ & $\begin{array}{c}2 \\
(1-5)\end{array}$ & $\begin{array}{c}04,58 b \\
(34,61) \\
\end{array}$ & $\begin{array}{c}1 \\
(1-2)\end{array}$ & $\begin{array}{c}12,03 a b c \\
(21,71)\end{array}$ & $\begin{array}{c}2 \\
(1-3)\end{array}$ & $\begin{array}{c}08,90 \mathrm{bc} \\
(32,14)\end{array}$ & $\begin{array}{c}2 \\
(1-4) \\
\end{array}$ \\
\hline 4 & $\begin{array}{c}09,37 \mathrm{bc} \\
(16,65)\end{array}$ & $\begin{array}{c}2 \\
(1-3)\end{array}$ & $\begin{array}{c}04,04 \mathrm{bc} \\
(32,67)\end{array}$ & $\begin{array}{c}2 \\
(1-2)\end{array}$ & $\begin{array}{c}13,86 \mathrm{ab} \\
(31,54)\end{array}$ & $\begin{array}{c}2 \\
(1-3)\end{array}$ & $\begin{array}{c}12,01 \mathrm{ab} \\
(18,66)\end{array}$ & $\begin{array}{c}1 \\
(1-5)\end{array}$ \\
\hline 5 & $\begin{array}{c}06,60 \mathrm{~cd} \\
(17,68)\end{array}$ & $\begin{array}{c}1 \\
(1-1)\end{array}$ & $\begin{array}{c}06,75 \mathrm{~b} \\
(35,22)\end{array}$ & $\begin{array}{c}1 \\
(1-1)\end{array}$ & $\begin{array}{c}08,43 \mathrm{~cd} \\
(28,19)\end{array}$ & $\begin{array}{c}1 \\
(1-1)\end{array}$ & $\begin{array}{c}\text { 06,50cde } \\
(27,52)\end{array}$ & $\begin{array}{c}1 \\
(1-1)\end{array}$ \\
\hline 6 & $\begin{array}{c}06,88 \mathrm{~cd} \\
(21,75)\end{array}$ & $\begin{array}{c}1 \\
(1-1)\end{array}$ & $\begin{array}{c}08,89 a b \\
(33,28)\end{array}$ & $\begin{array}{c}1 \\
(1-2)\end{array}$ & $\begin{array}{l}10,55 \mathrm{bc} \\
(26,95)\end{array}$ & $\begin{array}{c}1 \\
(1-1)\end{array}$ & $\begin{array}{c}08,63 \mathrm{bcd} \\
(33,88)\end{array}$ & $\begin{array}{c}1 \\
(1-1)\end{array}$ \\
\hline 7 & $\begin{array}{c}18,57 a \\
(18,16)\end{array}$ & $\begin{array}{c}3 \\
(1-3)\end{array}$ & $\begin{array}{c}14,94^{a} \\
(20,61)\end{array}$ & $\begin{array}{c}2 \\
(1-3)\end{array}$ & $\begin{array}{l}15,78 a \\
(19,02)\end{array}$ & $\begin{array}{c}3 \\
(1-6)\end{array}$ & $\begin{array}{c}17,50 a \\
(27,15)\end{array}$ & $\begin{array}{c}2 \\
(1-3) \\
\end{array}$ \\
\hline 8 & $\begin{array}{c}15,26 a b \\
(20,24)\end{array}$ & $\begin{array}{c}2 \\
(1-3)\end{array}$ & $\begin{array}{c}13,24 a \\
(20,19)\end{array}$ & $\begin{array}{c}2 \\
(2-3)\end{array}$ & $\begin{array}{c}12,59 \mathrm{abc} \\
(22,98)\end{array}$ & $\begin{array}{c}3 \\
(2-4)\end{array}$ & $\begin{array}{c}15,88 a \\
(30,21)\end{array}$ & $\begin{array}{c}2 \\
(1-3)\end{array}$ \\
\hline
\end{tabular}

Trat.: tratamento, $\mathrm{PI}$ : pré-tratamento seco, P2: pré-tratamento úmido, P3: pré-tratamento alta temperatura, P4: pré-tratamento elevada temperatura e umidade. Médias de pré-tratamentos representam módulo de ruptura (MOR) expresso em MPa; Valores entre parênteses nos pré-tratamentos representam o coeficiente de variação em porcentagem; FM: nível de classificação de porcentagem de falha na madeira. Valores entre parênteses no FM representam os níveis mínimos e máximos. Médias seguidas pela mesma letra na coluna não diferem estatisticamente entre si pelo teste de Friedman ao nível de $95 \%$ de probabilidade.

Os valores médios de tensão máxima na condição seca (P1) demonstraram que somente os tratamentos compostos por adesivo EPI e junções finger-joint (T7 e T8) atenderam ao requisito mínimo de 13,8 MPa para resistência e de percentual de falha na madeira (FM), em função de apresentar medias superiores à 60\% como determinado pela norma ASTM D5572 (ASTM, 2005).

Outro ponto a ser destacado é a ausência da diferença estatística entre as duas gramaturas de cada adesivo e tipo de junção, T1 e T2, T3 e T4, T5 e T6, e entre T7 e T8. Este comportamento corrobora com Follrich et al. (2010), que descrevem que o pequeno aumento na gramatura de peças (180 para $200 \mathrm{~g} / \mathrm{m}^{2}$ ) coladas não demonstra aumento significativo para a resistência ao teste de tração, principalmente em adesivos PVA.

Assim, pode-se afirmar que o aumento da gramatura não representa necessariamente uma maior aderência à madeira, pois o adesivo PVA apresenta baixa penetrabilidade na estrutura da parede celular, sendo acumulado na superfície da madeira. Esta característica mostra-se benéfica a peças coladas submetidas ao esforço de flexão, conferindo maior elasticidade à linha de cola e auxiliando na distribuição uniforme das tensões, contudo, em esforços de tração a junção apresenta menor eficiência de colagem, principalmente pela menor ancoragem do adesivo na madeira (FOLLRICH et al., 2010; CLAU et al., 2011).

Os tratamentos produzidos com madeira de C. lusitanica na condição seca, independente da composição foram inferiores aos obtidos por Prata (2010) utilizando adesivo PVA em madeira de P. taeda $(24,54 \mathrm{MPa})$, P. chiapensis $(24,54 \mathrm{MPa})$, P. caribaea var. hondurensis $(30,37 \mathrm{MPa})$, P. maximinoi $(27,42 \mathrm{MPa}), P$. oocarpa $(27,63 \mathrm{MPa})$ e P. tecunumanii $(29,46 \mathrm{MPa})$. Contudo cabe ressaltar que a maior geometria de junção finger-joint utilizada pelo autor citado, confere maior resistência 
ao esforço de tração.Para a condição úmida (P2) apenas os tratamentos T7 (EPI, 180, finger), T8 (EPI, 200, finger) e T6 (EPI, 200, plana) atenderam ao recomendado pela norma (6,9MPa). Já os demais, apresentaram acentuada redução da resistência causada pela degradação da linha de cola pela ação da água. Contudo quando considerada o percentual de falha na madeira (FM), apenas os tratamentos T4 (PVA, 200, finger), T7 (EPI, 180, finger) e T8 (EPI, 200, finger) atenderam os requisitos da norma.

Em estudo com espécies de pinus tropicais, Prata (2010) obteve valores muito superiores aos obtidos no presente estudo, P. taeda $(27,85 \mathrm{MPa})$, P. chiapensis $(30,48 \mathrm{MPa})$, P. caribaea var. hondurensis $(38,21 \mathrm{MPa})$, P. maximinoi $(36,26 \mathrm{MPa})$, P. oocarpa $(30,59 \mathrm{MPa})$ e P. tecunumanii $(39,23 \mathrm{MPa})$.

Os resultados obtidos para o adesivo PVA e junção de topo plana em condição de alta temperatura (P3) corroboram com o comportamento apresentado para o ensaio de flexão estática e ao descrito por Clau et al. (2011), que descrevem que o adesivo PVA apresenta degradação térmica a partir de $50^{\circ} \mathrm{C}$. Assim, as uniões planas além de apresentarem médias inferiores, também não atingiram o requisito descrito pela norma $(6,9 \mathrm{MPa})$, em virtude de serem consideradas mais sensíveis à degradação térmica. Contudo os ensaios demonstraram que para as uniões de topo tipo finger-joint, não existem diferenças estatísticas suficientes para suportar tal afirmação. Os tratamentos T3 (PVA, 180 finger), T4 (PVA, 200, finger), T7 (EPI, 180, finger) e T8 (EPI, 200, finger), atenderam à norma ASTM D5572 (ASTM, 2005), contudo as médias obtidas foram inferiores às obtidas por Prata (2010) para P. taeda (24,54 MPa), P. chiapensis $(26,05 \mathrm{MPa})$, P. caribaea var. hondurensis $(30,37 \mathrm{MPa})$, P. maximinoi $(27,42 \mathrm{MPa})$, P. oocarpa $(27,63 \mathrm{MPa})$ e P. tecunumanii $(29,46 \mathrm{MPa})$.

$\mathrm{O}$ ensaio de tração realizado em condição de elevada temperatura e umidade (P4) foi comparado somente à norma ASTM 5572 (ASTM, 2005), devido à falta de artigos em periódicos para comparação. A referida norma descreve que os tratamentos devem apresentar resistência mínima de 5,2 MPa, o que não foi atendido apenas nos tratamentos T1 (PVA, 180, plana) e T2 (PVA, 200, plana). Entre os demais tratamentos destacam-se como superiores os tratamentos T7 $(17,50 \mathrm{MPa})$, T8 (15,88 MPa) e T4 (12,01 MPa), destacando o fator de composição com junção do tipo finger-joint como a mais influente para a resistência à tração.Para o tratamento T4 a influência da maior gramatura deve ser destacada, haja vista, que a maior quantidade de adesivo na interface madeira-adesivo permite uma maior ancoragem das peças coladas e consequentemente maior resistência às tensões aplicadas mesmo em condições não favoráveis ao tipo de adesivo utilizado.

Assim, considerando os resultados obtidos para os ensaios de flexão estática e tração, independente do adesivo e do pré-tratamento aplicado, o fator gramatura não demonstrou influência suficiente para ser observada diferença estatística, o que para estudos futuros, faz-se necessária uma análise exploratória mais aprofundada da influência dos fatores componentes.Contudo, a origem da falta de variação pode ser decorrente de inúmeros fatores, dentre os quais podemos citar, a elevada pressão aplicada durante a colagem das peças e consequente escorrimento do adesivo, a textura fina da madeira de C. lusitanica dificultar a penetração do adesivo de forma ideal, além da indisponibilidade de equipamentos adequados para a produção de painéis colados lateralmente.

\section{CONCLUSÕES}

Os valores médios encontrados para resistência máxima nos ensaios de flexão estática e tração paralela às fibras permitem afirmar que os melhores tratamentos foram o T7 (gramatura $180 \mathrm{~g} / \mathrm{m}^{2}$ ) e o T8 (gramatura $200 \mathrm{~g} / \mathrm{m}^{2}$ ), ambos compostos por adesivo EPI e junção de topo tipo finger-joint, assim como para porcentagem de falhas na madeira. Quanto aos parâmetros da ASTM D5572 (ASTM, 2005), observou-se que somente os tratamentos T7 e T8 atenderam aos requisitos mínimos, diferenciando dos demais principalmente quanto ao percentual superior a $60 \%$ de falhas na madeira.

O comportamento das junções com adesivo PVA foi comprometida com a aplicação dos pré-tratamentos, especialmente quanto exposto a condições de umidade e temperatura elevadas, o que prejudicou os resultados. 
Almeida et al. - Avaliação da qualidade da colagem de topo da madeira de Cupressus lusitanica Mill. para a produção de painéis colados lateralmente

\section{REFERÊNCIAS BIBLIOGRÁFICAS}

ABNT - ASSOCIAÇÃO BRASILEIRA DE NORMAS TÉCNICAS. NBR 7190 - Projeto de estruturas de madeira. Rio de Janeiro, 1997. 107 p.

ASTM - AMERICAN SOCIETY FOR TESTING AND MATERIAL. D5572 - Standard Specification for Adhesives Used for Finger joints in Nonstructural Lumber Products. Philadelphia, 2005. 17 p.

CARNEIRO, M. E.; BITTENCOURT, E.; MUNIZ, G. I. B. Qualidade da madeira de Cryptomeria japonica D. Don. Floresta, Curitiba, v. 39, n. 4, p. 913-920, 2009.

CHAVES, E.; FONSECA, W. Ciprés. (Cupressus lusitanica). Especie de árbol de uso múltiple en América Central. Colección de guías silviculturales. Turrialba: Catie, 1991. 70 p. (Serie técnica. Informe técnico, 168).

CLAUß, S.; JOSCAK, M.; NIEMZ, P. Thermal stability of glued wood joints measured by shear tests. European Journal of Wood and Wood Products, v. 69, n. 1, p.101-111, 2011.

FOLLRICH, J.; TEISCHINGER, A.; MÜLLER, U. Artificial ageing of softwood joints and its effect on internal bond strength with special consideration of flat to end grain joints. European Journal of Wood and Wood Products, v. 69, n. 4, p. 597-604, 2011.

FOLLRICH, J.; VAY, O.; VEIGEL, S.; MÜLLER, U. Bond strength of end-grain joints and its dependence on surface roughness and adhesive spread. Journal Wood Science, v. 56, n. 5, p. 429-434, 2010.

GALINARI, R.; TEIXEIRA JUNIOR, J. R.; MORGADO, R. R.; A competitividade da indústria de móveis do Brasil: situação atual e perspectivas. Bens de Consumo BNDES Setorial, Rio de Janeiro, n. 37, p. 227-272, 2012.

IWAKIRI, S.; VIANEZ, B. F.; WEBER, C.; TRIANOSKI, R.; ALMEIDA, V. C.; Avaliação das propriedades de painéis aglomerados produzidos com resíduos de serrarias de nove espécies de madeiras tropicais da Amazônia. Acta Amazonica, Manaus, v. 42, n. 1, p. 59-64, 2012a.

IWAKIRI, S.; MATOS, J. L. M.; TRIANOSKI, R.; PRATA, J. G.; Produção de painéis aglomerados homogêneos e multicamadas de Melia azedarach (Cinamomo) e Pinus taeda com diferentes teores de resina. Cerne, Lavras, v.18, n.3, p.465-470, 2012b.

OKINO, E.Y.A.; SANTANA, M. A. E.; ALVES, M.V.; MELO, J.E.; CORADIN, V.T.R.; SOUZA, M.R.; TEIXEIRA, D.E.; SOUSA, M.E. Technological Characterization of Cupressus spp. Wood. Floresta e Ambiente, Seropédica, v. 17, n. 1, p. 1-11, 2010.

OKINO, E. Y. A.; TEIXEIRA, D. E.; SOUZA, M. R.; SANTANA, M. A. E.; SOUSA M. E. Propriedades de chapas OSB de Eucalyptus grandis e de Cupressus glauca. Scientia Forestalis, Piracicaba, v. 36, n. 78, p. 123-131, 2008.

PEREIRA, J. C. D.; HIGA, R. C. V. Propriedades da Madeira de Cupressus lusitanica Mill. Embrapa Florestas Comunicado técnico, Colombo, n. 107, 5 p. 2003.

PINTO. P. H. M. A.; NOLASCO. A. M.; ULIANA. L. R. Estratégias para redução da geração de resíduos em indústrias de piso de madeira. SIMPÓSIO DE INICIAÇÃO CIENTÍFICA DA USP, 2012, Piracicaba. Anais... Piracicaba: USP, 2012. Disponível em: < http://www.pimads.org/documento atividades/Poster\%2003\%20 -\%20SIICUSP>. Acesso em: 05 jul. 2015.

PRATA, J. G.; Estudo da viabilidade tecnológica do uso de espécies de pinus tropicais para produção de painéis colados lateralmente (Edge Glued Panels - EGP). 117 p. 2010. Tese (Doutorado em Engenharia Florestal) - Universidade Federal do Paraná, Curitiba, 2010. 
ROQUE, R. M.; GARITA, C. S.; ZÚÑ̃IGA, L. L.; Ciprés: Cupressus lusitanica Mill. Cupressaceae. Ficha técnica 4. Tecnología de madera de plantaciones forestales. Revista Forestal Mesoamericana, Kurú, v. 7, n. 18-19, p. 76-90, 2010.

SHIMIZU, J. Y.; JUNIOR, U. R.; CANCELA, K. C.; MAIOCHI R. A. Cedrinho como alternativa para produção de madeira em pequenas propriedades rurais. Embrapa Florestas Comunicado Técnico, Colombo, n.17, 23 p., 2006.

SHIMIZU, J. Y.; PINTO JÚNIOR, J. E.; RIBASKI, G. Cipreste para madeira: alto incremento volumétrico com material genético apropriado. Boletim de Pesquisa Florestal, Colombo, n.30/31, p.3-17, 1995.

TRIANOSKI, R.; IWAKIRI, S.; MATOS, J. L. B. Avaliação de painéis aglomerados de Toona ciliata produzidos com diferentes densidades e teores de resina. Madera y Bosques, Xalapa, v. 20, n. 3, p. 49-58, 2014.

TRIANOSKI, R.; IWAKIRI, S.; MATOS, J. L. M.; CHIES, D. Utilização da madeira de Cryptomeria japonica para produção de painéis aglomerados. Scientia Forestalis, Piracicaba, v. 41, n. 97, p. 57-64, 2013.

VICK, C. B. Adhesive Bonding of Wood Materials - Wood handbook - Wood as an engineering material. Madison: Department of Agriculture, 1999. 463 p. (Gen. Tech. Rep. FPL - GTR -113).

VRAZEL, M.; SELLERS JR, T. The effects of species, adhesive type, and cure temperature on the strength and durability of a structural finger-joint. Forest Product Journal, Madison, v. 54, n. 3, p. 66-75, 2004.

XAVIER, S. A.; FUKAMI, J.; MIOTTO, L. C. V.; SOBOTTKA, R. P.; NAKATANI, S. H.; TAKAHASHI, L. S. A.; MACHADO, M. H. Superação da dormência de sementes de Cupressus lusitanica Mill. Semina: Ciências Agrárias, Londrina, v. 33, n. 3, p. 1041-1046, 2012.

Recebido em 08/09/2015

Aceito para publicação em 27/05/2016

Sci. For., Piracicaba, v. 45, n. 113, p. 9-19, mar. 2017

DOI: dx.doi.org/10.18671/scifor.v45n113.01 